\title{
Adhesion strength of protective and decorative coatings depending on the porosity of the cement substrate
}

\author{
Valentina Loganina ${ }^{l, *}$, and Bassam Shareef Denied Al Saedi ${ }^{2}$ \\ ${ }^{1}$ Penza State University of Architecture and Construction" Penza, German Titov str., 28, 440028, \\ Russia \\ ${ }^{2}$ Chief Engineer of the South Rifineriers Company,Basra,Ministry of Oil,Irag
}

\begin{abstract}
Information provided about long-term bonding strength protective decorative coatings. A method is proposed for assessing the longterm bonding strength of coatings. The method consists in determining the bonding strength before testing and after $t$ hours of testing. The dependence of the long-term bonding strength on the porosity of the cement substrate has been established. With an increase in the surface porosity of the substrate, observed a decrease in the time of maintaining the adhesive contact "coating-substrate». The aging process of coatings is considered from the position accumulation of damage, taking into account the hereditary factor. The influence of the hereditary factor on the kinetics of damage accumulation was established. It is shown that the dependence of the operating time on the level of damage accumulation can be approximated by an exponential dependence. The values of the activation energy of adhesive destruction of coatings are calculated.
\end{abstract}

\section{Introduction}

The operational durability of protective and decorative coatings of external walls of buildings is significantly influenced by the processes occurring both in the coating itself and at the "substrate-coating" contact boundary [1,2]. The adhesion strength of protective and decorative coatings to a concrete substrate substantially depends on the quality of the substrate. The quality of the substrate, first of all, is understood as its macro- and microstructure, the degree of its homogeneity, ensuring the desired solidity of the contact layer, its density and porosity. Features of a porous substrate, such as cement concrete, mortar, etc., have a significant impact on the formation of the structure and properties of the applied coatings. The surface layer of the substrate has some differences from the base material. It is characterized by increased capillary porosity and a lower value of physical and mechanical properties. The presence of a weakened surface layer of the substrate creates the preconditions for a lower operational resistance of protective and decorative coatings [3-6].

\footnotetext{
* Corresponding author: loganin@mail.ru
} 


\section{Materials and research methods}

In order to determine the flaking probabilities of the studied coverage, tests were carried out on colored samples with different porosity of the substrate ( 20 samples for each porosity) to determine the change in bonding strength during successive wetting and drying of coatings. Paints were applied to the substrate in two layers with intermediate drying for 20 minutes. Polyvinyl acetatcement, lime, organosilicon KO-168 paints were used as paint compositions. In this case, the porosity of the substrate was varied from $0 \%$ to $40 \%$.

The parameters of strength and destruction of coatings were evaluated as follows. According to the temperature-time dependence, the strength of polymer coatings can be described by the equation[7-9]

$$
\tau=\tau_{0} e \frac{U_{0}-\gamma \sigma}{R T}
$$

where $\tau_{0}$ is a constant numerically equal to the period of vibrations of atoms;

$U_{0}$ - initial activation energy of destruction;

$\gamma$-structure-sensitive factor characterizing the overvoltage of bonds in the polymer;

$\sigma$ - applied voltage;

$R$ - gas constant;

$T$ - temperature, ${ }^{\circ} \mathrm{K}$.

The activation energy of adhesive destruction was determined by the express method. The method consists in determining the bonding strength before testing and after t hours of testing. Graphical dependence of the adhesion strength in the aging process in a semi logarithmic coordinate system is approximated by an equation of the form:

$$
\lg R_{a d}^{t}=\lg R_{a d}^{0}-k
$$

where $\mathrm{t}$ is the aging time;

$U_{0}$ - initial adhesion strength value.

The value of adhesion strength after t hours of operation.

$$
\frac{\lg R_{a d}^{0}}{\lg R_{a d}^{0}-\lg R_{a d}^{t}}=\frac{\tau}{t}
$$

where t-current value of aging time

After substituting the equation (3) into the equation (1), we obtain

$$
\frac{\lg R_{a d}^{0}}{l g R_{a d}^{0}-l g R_{a d}^{t}}=\frac{\left[\tau_{0} \exp \left(\frac{U}{R T}\right)\right.}{t}
$$

Equation (4) makes it possible to estimate the value of the activation energy of the destruction of adhesive bonds based on the results of testing the bonding strength $R_{a d}^{0}, R_{a d}^{t}$

$$
U=R T \ln \left[\frac{\lg R_{a d}^{0} t}{\left(\lg R_{a d}^{0}-\lg R_{a d}^{t}\right) \tau_{0}}\right]
$$

To assess the adhesion strength, we used the method tearing off the stamp (normal separation) according to GOST 32299-2013 «Materials are paint and varnish. Determination of adhesion by the pull-off method». The method consists in measuring the force required to lift the coating off the concrete surface with help glued metal stamp cylindrical shape with a diameter of $18 \mathrm{~mm}$. The pull-off force was measured using a dynamometer. To glue the stamps to the coating, we used epoxy glue (EDP-TU 0751-018-48284381-00). The adhesion strength of the paint to the substrate, $\mathrm{MPa}$, was determined by the formula:

$$
R_{a d}=\frac{P}{F}
$$


where $P$ is the separation force, $\mathrm{N}$;

$F$ - contact area of the stamp with the paint-and-lacquer coating, $\mathrm{m}^{2}$.

\section{Research results}

It is known that the dependence of the adhesion strength to detachment $R$ on the size of the contact area $S$ is expressed by the relation

$$
R_{a d}=c S^{-1 / n}
$$

where $c, n$ are the constants taking into account the distribution of stresses and defects in the sample.

Investigation of the structure of the "coating-substrate" contact layer showed that there are pores in the contact zone that are not filled with the paint composition. In accordance with the theory of percolation, a decrease in the strength of a composite material with an increase in its porosity occurs according to the law

$$
R_{a d}=R_{0}\left(1-\frac{P}{0.84}\right)^{d}
$$

where $\mathrm{R}_{0}$ is the strength of the pore-free composite;

$\mathrm{d}$ is the universal critical index.

As a result of mathematical processing of the experimental data shown in Fig. 1, it was found that the value of $d$ is $=0.39$. In accordance with the theory of percolation, this means that the strength of the composite contact layer is influenced by the entire array of structural bonds. For PVAC coating, the strength of the defect-free contact layer is $\mathrm{R} 0=2.8 \mathrm{MPa}$, for organosilicon KO-168 - 2.5 MPa. The presence of pores in the contact zone promotes premature flaking of coatings.

Consider the aging process of coatings from the perspective of damage accumulation, taking into account the hereditary factor. Let us estimate the degree of damage to the material by the level of damage accumulation $\mathrm{W}$, which can be determined by the formula:

$$
W=\frac{R_{0}-R_{d}}{R_{0}}
$$

where $R_{d}$ is the actual bond strength;

$R_{0}$ - adhesion strength of a defect-free coating

Fig. 1 shows the values of the level of damage accumulation during the test. $\mathrm{W}_{0}$ is a hereditary factor that has a significant impact on the kinetics of damage accumulation. An analysis of the experimental data (Fig. 1) indicates that at the beginning of the aging process (incubation period), there is a slight change in the level of damage accumulation. The duration of the incubation period depends on the level of the hereditary factor $\mathrm{W}_{0}$. The higher the initial level of damage accumulation $\mathrm{W}_{0}$, the faster the intensive accumulation of damage begins. With a porosity of the cement substrate $\mathrm{P}=12.3 \% \mathrm{~W}_{0}=0.04$, the incubation period is 25 cycles, and with a porosity of $\mathrm{P}=23 \% \mathrm{~W}_{0}=0.12$ the incubation period is equal to 15 cycles. Accordingly, the rate of damage growth for a coating on a substrate with a porosity of $12.3 \%$ is 0.25 , and on a substrate with $\mathrm{P}=23 \%-0.37$. At $\mathrm{W}=0.85$, the coatings are destroyed.

An analysis of the experimental data (Fig. 2) indicates that at the beginning of the aging process (incubation period), there is a slight change in the level of damage accumulation. The duration of the incubation period depends on the level of the hereditary factor $\mathrm{W}_{0}$. The higher the initial level of damage accumulation $\mathrm{W}_{0}$, the faster the intensive accumulation of damage begins. With a porosity of the cement substrate $\mathrm{P}=12.3 \% \mathrm{~W} 0=0.04$, the incubation period is 25 cycles, and with a porosity of $\mathrm{P}=23 \% \mathrm{~W} 0=0.12$ the incubation period is equal to 15 
cycles. Accordingly, the rate of damage growth for a coating on a substrate with a porosity of $12.3 \%$ is 0.25 , and on a substrate with $\mathrm{P}=23 \%-0.37$. At $\mathrm{W}=0.85$, the coatings are destroyed.

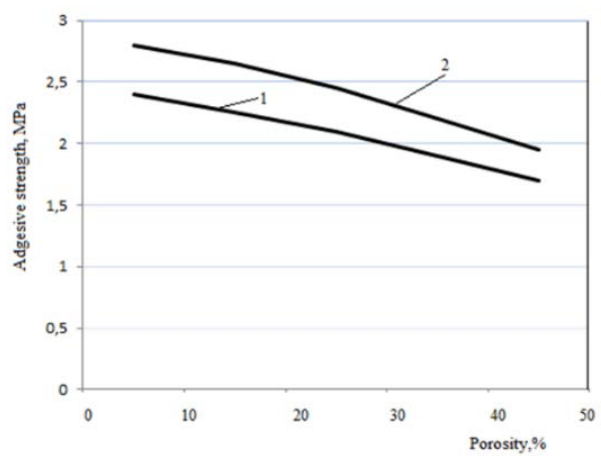

Fig. 1. Dependence of the adhesion strength of coatings on the porosity of the substrate 1 - KO-168; 2 - coating PVAC

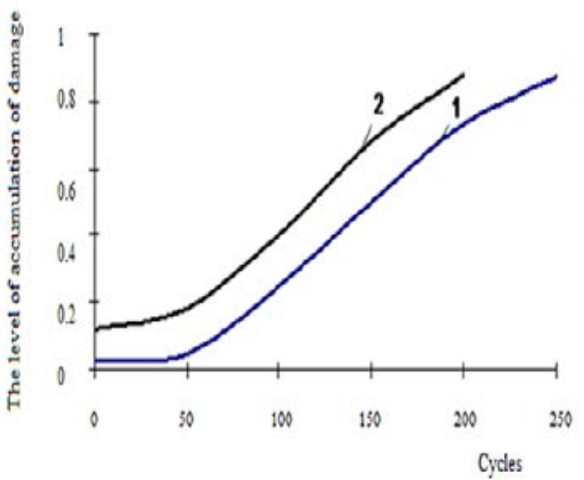

Fig. 2. Change in the level of accumulation of damage to the coating of KO-168 during aging: 1 - coating on a substrate with a porosity of $12.3 \%$;

2 - coating on a substrate with a porosity of $23 \%$.

Mathematical processing of the data shown in Fig. 2 shows that the dependence of the operating time on the level of damage accumulation can be approximated by an equation of the form:

$$
\tau=a \exp (b \ln w)
$$

For the organosilicon KO-168 coating on a substrate with a porosity of $12.3 \%$, equation (10) has the form

$$
\tau=259.8 \exp (0.6856 \ln w)
$$

On a substrate with $\mathrm{P}=23 \%$

$$
\tau=254.95 \exp (1.1848 \ln w)
$$

Equation (10) makes it possible to estimate the operating time of the coating at a critical level of damage accumulation, i.e. at the moment of "failure". Undoubtedly, the dependence (10) reflecting the duration of bonding strength in time depending on the initial level of damage accumulation, is more complex. In accordance with the kinetic concept of the strength of solids, the dependence of the duration of retention of adhesive strength takes the form

$$
\tau=\tau_{0} \exp \frac{\left(U^{a d}-\gamma \sigma\right)}{R T}
$$

where $U^{\text {ad }}$ is the initial activation energy of adhesive destruction;

$\gamma$ - structure-sensitive coefficient;

$R$ is the Boltzmann constant;

$T$ - temperature, $\mathrm{K}$;

$\sigma$-applied stress.

However, in the formula (1) the values $\tau_{0}, \gamma, \mathrm{U}$ are effective quantities that characterize not an elementary reaction, but a complex combination of several elementary reactions and ongoing phase and structural transformations. When estimating the parameters of equation 
(1), certain difficulties are caused by taking into account internal stresses and their variable value due to relaxation processes. In addition, in accordance with the equation (1), the life curves for different temperatures should converge at one pole, however, numerous works indicate that for adhesive joints a single pole is not observed [10,11]. According to the author [12], with increasing temperature there is an increase in the coefficient. According to the author $[13,14]$, with increasing temperature there is an increase in the coefficient $\gamma$. This is due to a change in the ratio of the processes of destruction and relaxation.

In Fig. 3 the dependence of the long-term adhesion strength of coatings on stresses in a semilogarithmic coordinate system is given. Experimental data have confirmed that the duration of preservation of the bonding strength of cement concrete coatings can be described by an exponential relationship.

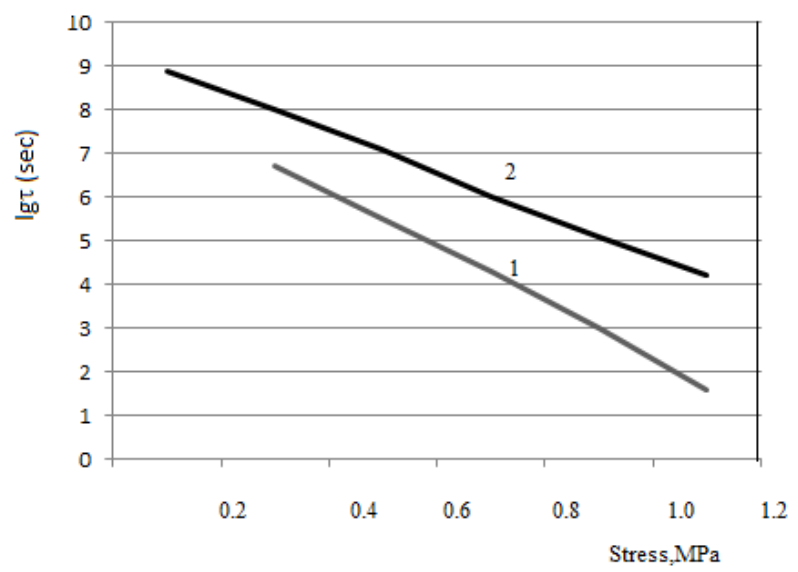

Fig. 3. Long-term adhesion of coatings: 1 -coating PVAC; 2 - coating KO-168

It was found that under the action of a constant voltage there is a proportional relationship between the logarithm of the time of adhesive destruction of coatings and the index of the surface porosity of the substrate (Fig.4). With an increase in the surface porosity of the substrate, is observed reducing the time of maintaining the bonding contact "coatingsubstrate". The data obtained indicate the statistical nature of the adhesion strength under static fatigue and the significant role of defects in the process of destruction of adhesive joints. The dependence of the long-term adhesion strength on the surface quality of the cement substrate can be described by an equation of the form

$$
\tau=\operatorname{Bexp}(-k P)
$$

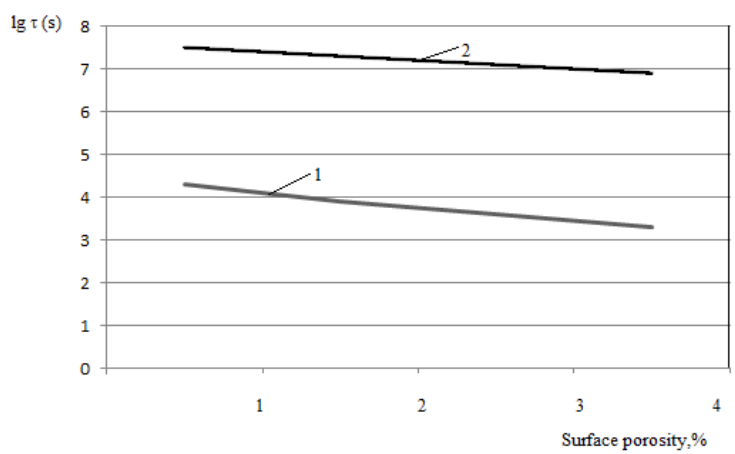

Fig. 4. Long term bond strength depending on the porosity of the substrate: 1 -coating PVAC; 2 coating KO-168 
For PVAC coating, equation (14) has the form

$$
\tau=1698.24 \exp (-0.81 P)
$$

For coatings KO-168

$$
\tau=1698.24 \exp (-0.58 P)
$$

Considering that the long-term adhesion strength depending on the acting stress is expressed by the equation (13) the generalized equation of the long-term bond strength has the form

$$
\tau=\exp [-(\alpha P+\beta \sigma)]
$$

The expression $[-(\alpha \mathrm{P}+\beta \sigma)]$ is essentially the value of the local stress in the defect regions of the contact layer structure. Comparative analysis of formulas (13) and (14) indicates that they essentially reflect the duration of the retention of adhesion strength, taking into account the defectiveness of the contact layer, i.e.

$$
\tau=\tau_{0} \exp \frac{\left(U_{0}-\Delta U\right)}{R T}
$$

where $\Delta U=U_{d}+U$ excitation energy of joint action of defectiveness and stress.

The excitation energy due to defectiveness $U_{d}$ depends on the porosity of the cement substrate and the level of damage accumulation. The structure-sensitive coefficient characterizing bond overstress increases with increasing porosity, i.e. the defectiveness of the contact layer structure increases. So, for example, at a surface porosity of the substrate of $5 \%$ $\gamma=15.807 \mathrm{~kJ} / \mathrm{mol} \mathrm{MPa}$ and at $\mathrm{P}=7 \%-\gamma=17.023 \mathrm{~kJ} / \mathrm{mol} \mathrm{MPa}$. The values of the activation energy for the destruction of adhesive bonds for the coatings under study were calculated (Table 1)

Table 1. Values of activation energy for destruction of adhesive bonds of coatings

\begin{tabular}{|l|l|l|}
\hline Coating type & Aging time, cycles & Activation energy, kJ / mol \\
\hline Polyvinyl acetate cement & 20 & 26,731 \\
& 40 & 26.790 \\
& 60 & 26.832 \\
& 80 & 26.856 \\
& 100 & 26.901 \\
\hline Organosilicon KO-168 & 20 & 26,935 \\
& 40 & 27.019 \\
& 60 & 27.144 \\
& 80 & 27.208 \\
& 100 & 27.288 \\
\hline Lime & 20 & 25,759 \\
& 40 & 25.766 \\
& 60 & 25.899 \\
& 80 & 25.966 \\
& 100 & 26.063 \\
\hline
\end{tabular}

\section{Conclusions}

It has been established that the long-term adhesive strength of coatings depends on the porosity of the cement. It has been revealed that the kinetics of changes in the bond strength is influenced by the preliminary level of damage accumulation. A method is proposed for determining the activation energy of adhesive destruction. 


\section{References}

1. L. P. Orentlicher, V. I. Loganina, Protective decorative coatings for concrete and stone buildings ( Study guide, Moscow, Stroyizdat, 1992).

2. V.I.Loganina,.Increasing the Durability of Paint and Varnish Coatings in Building Products and Construction. https://www.elsevier.com/books/increasing-the-durabilityof-paint-and-varnish-coatings-in-building-products-and-construction/ivanovna/978-012-817046-5 (2019)

3. V.I.Loganina,Jury Skachkov, Assessment of the Stress State of the Coating in Depending on the Porosity of the Cement Substrate. Key Engineering Materials: Engineering Materials and Technology 737,179-183 (2016)

4. V.I.Loganina, J.P. Skachkov, The Application Of The Holographic Method For Evaluation Of A Stress Deformation State Of Cement Paint Coatings. International Journal of Applied Engineering Research. 11(14), 8377-8378 (2016)

5. L.A. Sukhareva, The durability of coatings ( Moscow, Chemistry, 1984)

6. P.I.Zubov, L.A.Suhareva, Structure and properties of polymer pokrytiy (Moscow, Chemistry, 1982)

7. S.N.Zhurkov, Time dependence of solids. Journal of technical physics, 1677 (1953)

8. S.N.Zhurkov, E.E. Tomashevsky, Study of the strength of solids . Journal of technical physics,66 (1955)

9. S.N. Zhurkov, T.P. Sanrirov, Temperature-time dependence of the strength of pure metals. Reports of the USSR Academy of Sciences, (1955) .

10. A.S. Freidin, Strength and durability of adhesive joints ( Moscow, Chemistry, 1982)

11. A.S. Freidin, R.A. Turusov, Properties and calculation of adhesive compounds (Moscow, Chemistry, 1990).

12. G.M .Bartenev, Yu.S.Zuev, Strength And destruction of highly elastic materials (Moscow-Leningrad, Chemistry, 1984)

13. Yu.N.Rabotnov, Mechanics of a deformable solid (Moscow: Science,1988)

14. I.E. Prokopovich, V.A. Zedgenidze, Applied Creep Theory (Moscow:Stroyizdat,1980) 